\title{
Exercise-Induced Oxidative Stress and Dietary Antioxidants
}

\author{
Abbas Yavari ${ }^{1}$; Maryam Javadi ${ }^{2}$; Parvin Mirmiran ${ }^{3,}$; Zahra Bahadoran $^{3}$ \\ ${ }^{1}$ Department of Physical Education, Tabriz University of Medical Sciences, Tabriz, IR Iran \\ 2 Department of Nutrition, Faculty of Health, Qazvin University of Medical Sciences, Qazvin, IR Iran \\ ${ }^{3}$ Department of Clinical Nutrition and Dietetics, Faculty of Nutrition Sciences and Food Technology, National Nutrition and Food Technology Research Institute, Shahid Beheshti \\ University of Medical Sciences, Tehran, IR Iran \\ ${ }^{*}$ Corresponding author: Parvin Mirmiran, Department of Clinical Nutrition and Dietetics, Faculty of Nutrition Sciences and Food Technology, National Nutrition and Food Technol- \\ ogy Research Institute, Shahid Beheshti University of Medical Sciences, Tehran, IR Iran. Tel: +98-2122432500, E-mail: mirmiran@endocrine.ac.ir \\ Received: October 28, 2014; Accepted: December 22, 2014
}

\begin{abstract}
Context: Overproduction of reactive oxygen and nitrogen species during physical exercise, exercise induced oxidative stress and antioxidant supplementation is interesting and controversial concepts that have been considered during the past decades.

Evidence Acquisition: In this review, we aimed to summarize current evidence in relation to antioxidant supplementation outcomes during exercise and physical activity. For this aim, we obtained relevant articles through searches of the Medline and PubMed databases between 1980 to 2013. Although major studies have indicated that antioxidants could attenuate biomarkers of exercise-induced oxidative stress and the use of antioxidant supplement is a common phenomenon among athletes and physically active people, there are some doubts regarding the benefits of these.

Results: It seems that the best recommendations regarding antioxidants and exercise are having a balanced diet rich in natural antioxidants and phytochemicals.

Conclusions: Regular consumption of various fresh fruits and vegetables, whole grains, legumes and beans, sprouts and seeds is an effective and safe way to meet all antioxidant requirements in physically active persons and athletes.
\end{abstract}

Keywords: Exercise-Induced; Oxidative Stress; Reactive Oxygen Species; Vitamin C; Vitamin E; Glutathione; Antioxidant; Minerals

\section{Context}

Oxidative stress, an impaired balance between free radical production and the endogenous antioxidant defense system, mainly leads to accumulation of oxidative damage, activation of stress-sensitive signaling pathways and development of pathologic conditions such as cardiovascular disease, insulin resistance and metabolic syndrome $(1,2)$.

Various identified pathologic as well as non-pathologic conditions identified could accelerate the production of free radicals or undermine the antioxidant defense system. Regular physical activity as well as prolonged and intensive exercise in spite of various beneficial effects on health promotion and reduction of the risk of cardiovascular disease, cancer, osteoporosis, diabetes and other chronic disease, is considered one of the oxidative stressinduced inducing conditions. Exercise training is accompanied with induction of oxidative stress via over production overproduction of reactive oxygen species (ROS) including superoxide anion ( $\left.\mathrm{O}^{2-}\right)$, hydroxyl (OHHO.) and peroxyl radical (RO2.) (3). Increased generation of reactive nitrogen species including peroxynitrite, generated from nitric oxide, are also involved in exercise-induced oxidative stress (3).

For the first time, in 1978, exercise-induced oxidative stress in humans was reported (4) currently, there is a large growing body of literature regarding the origins of ROS generation in skeletal muscle, the functions and consequences of ROS production in muscle, and oxidative damage of macromolecules and their metabolites as well as antioxidant supplementation to attenuate this oxidative condition (4-6). In the recent years, despite many advances in the concept of exercise-induced oxidative stress, there is controversy in this regard; whereas older studies have mainly focused on the adverse effects of ROS generation in the muscles including muscle damage and dysfunction, recent investigations report that mitochondrial ROS production in the muscle during exercise is programmed and required for main signaling pathways for muscle adaptation to exercise $(3,7,8)$ recent researches even propose that some health-promoting effects of exercise are related to ROS generation and exercise-induced free radical production could promote insulin sensitivity and help to prevent type 2 diabetes $(9,10)$. Beyond this, exercise-induced ROS activates some redox-sensitive signaling pathways and modulates the endogenous antioxidant defense system $(11,12)$. In fact, both the positive and negative aspects of ROS generation in sport performance are currently considered (13). These inconsistencies may raise the question whether supplementation with antioxidants during exercise

Copyright ( 2015 , Sports Medicine Research Center. This is an open-access article distributed under the terms of the Creative Commons Attribution-NonCommercial 4.0 International License (http://creativecommons.org/licenses/by-nc/4.0/) which permits copy and redistribute the material just in noncommercial usages, provided the original work is properly cited. 
Yavari A et al.

training programs or regular physical activity has favorable or unfavorable effects or not.

In this review, we briefly described the possible mechanisms of exercise-induced oxidative stress, modulatory effects of exercise on the endogenous antioxidant system, and then summarize current evidence in relation to antioxidant supplementation outcomes during exercise and physical activity. For this aim, we obtained relevant articles through searches of the Medline and PubMed databases.

\section{Evidence Acquisition}

\subsection{Oxidative Stress}

Oxidative stress is a physiological pathway which has important roles in the body under normal conditions (14). Commonly, overproduction of reactive oxygen species (ROS) or a defect in endogenous antioxidant defense system, including enzymatic and non-enzymatic antioxidants, has been defined as oxidative stress (11). Reactive oxygen species include active molecular oxygen such as hydroxyl (HO.) and superoxide $\left(\mathrm{O}^{2}\right)$ radicals as well as hydrogen peroxide $\left(\mathrm{H}_{2} \mathrm{O}_{2}\right)$ Mitochondrion, peroxisomal oxidase, cytochrome P450, NADP $(\mathrm{H})$ oxidase and xanthine oxidase are main sources of ROS production (14). overproduction of ROS and oxidative stress contribute to development of oxidation of cellular macromolecules such as lipids, proteins and DNA which could lead to pathogenesis of various degenerative and chronic diseases (15).

\subsection{An Overview on Free Radicals Generation Dur- ing Physical Exercise}

During physical exercise, oxygen flux increases to active skeletal muscles, which leads to enhanced production of ROS and free radicals $(16,17)$. Exercise increases the oxygen uptake 10 to 20 fold and promotes generation of ROS and free radicals which attack biological macromolecules especially DNA, polyunsaturated fatty acids, amino acids and active proteins (18). Increased metabolic rate and consumption of oxygen by muscle fibers, increased temperature and decreased $\mathrm{PH}$ of cellular muscle during the exercise could accelerate the production of free radicals. There are some debates regarding the origins of the ROS production during the exercise, but the skeletal muscle has mostly been considered as the major source of ROS generation (4). Major sites of ROS generation in active muscles during exercise are mitochondria, xanthine oxidase, nicotinamide adenine dinucleotide phosphate (NADPH) oxidase, phospholipase A2 dependent processes, and some immune cells including macrophages, monocytes, eosinohpils and neutrophils $(3,5)$.

Increased levels of catecholamines, as well as increased release of metmyoglobin from damaged muscle and interaction of metmyoglobin and methaemoglobin with peroxides during exercise also have been proposed as mediators of ROS generation $(4,19)$.
As mentioned earlier, both harmful and beneficial effects of ROS and RNS generation during exercise training have been suggested.

\subsection{Modulatory Effects of Exercise on Endogenous Antioxidant Defense System}

Another controversial issue regarding exercise-induced oxidative stress is that free radicals generated during physical activity act as hormesis and activate some important components of the endogenous antioxidant defense system such as superoxide dismutase and glutathione peroxidase, in other words, physical activity especially non-exhaustive moderate exercise may act as the best antioxidant $(11,12)$. Acute and chronic physical activity have a different effect on oxidative stress; studies showed that acute exercise induces reactive oxygen and nitrogen species and oxidative stress, but regular exercise training induces the endogenous antioxidative system and protects the body against adverse effects of oxidative damage (20). Moreover, different organs also have various responses to oxidative stress induced by acute and chronic exercise (21). Recent studies indicated that ROS generated during exercise are initiators of two important redox-sensitive signaling pathways including nuclear factor $\kappa \mathrm{B}(\mathrm{NF}-\kappa \mathrm{B})$ and mitogen activated protein kinase (MAPK) (22). Activation of these pathways leads to induction of antioxidant enzymes including mitochondrial superoxide dismutase (MN SOD) and glutathione peroxidase (GPX) as well as inducible nitric oxide synthase $(12,20)$. Evaluation of the long-term effects of exercise training showed that muscle content of reduced glutathione (GSH), a main substrate for GPX, increased and the glutathione disulfide ratio decreased during exercise training (23). Exercise training also has been accompanied with higher activity of catalase (24).

\subsection{Dietary Antioxidants and Exercise-Induced Oxidative Stress}

Several dietary antioxidants have been identified which could contribute to protection against free radicals production and oxidative damage, induction of antioxidant signaling pathways, promotion of the endogenous antioxidant defense system, attenuation of oxidative stress and consequently, prevention of related disorders (25). Currently, diets with high total antioxidant capacity and antioxidant-rich foods, are considered to be interesting approaches for prevention of several chronic diseases (cardiovascular disease, metabolic syndrome, many types of cancers, etc.), and management of some pathologic conditions such as type 2 diabetes, and insulin resistance (26-29).

Several functions have been investigated for antioxidants to protect against ROS-mediated injuries by both endogenous and induction of exogenous antioxidants. These functions include conversion of ROS into less active molecules and induction of some important anti- 
Yavari A et al.

oxidative enzymes (6). By For the reasons discussed in previous sections, the use of antioxidants with the aim to attenuate exercise-induced oxidative stress and related consequences is one of the controversial topics regarding application of dietary antioxidants especially in supplement form. Although major investigations have indicated that antioxidants could attenuate biomarkers of exercise-induced oxidative stress and the use of antioxidant and vitamin supplement is a common phenomenon among athletes and physically active people, there are however some doubts in relation to the advantages and disadvantages of these $(6,10)$ some investigations have reported beneficial effects but some others have indicated adverse outcomes following vitamin and antioxidant supplementation in athletes (30). In the following, more important antioxidants commonly used as supplement during exercise training and the outcomes of these are discussed.

\section{Results}

\subsection{Glutathione}

Glutathione is an endogenous Thiel group-containing antioxidant that reacts with ROS as a co-factor of the antioxidant enzyme glutathione peroxidase. Glutathione has several roles in the cellular antioxidant defense system via directly scavenging free radicals, and reducing or "recycling" various antioxidants (including vitamin $\mathrm{E}$ and vitamin $\mathrm{C}$ radicals) (31). $\mathrm{N}$-acetyl cysteine, a watersoluble precursor of glutathione, enhancing glutathione synthesis, is one of the antioxidant supplements used in exercise training (32). Supplementation with $200 \mathrm{mg} / \mathrm{d}$ $\mathrm{N}$-acetyl cysteine in untrained healthy men before incremental cycling prevented any increase in the capacity of neutrophils to generate ROS (33). In another study on rowers, $\mathrm{N}$-acetyl cysteine supplementation ( $6 \mathrm{~g}$ for three days before completing 6 min of maximal exercise) led to significant reduction of in vitro neutrophils ROS production below pre-exercise values (17). A seven-day administration of N-acetyl cysteine could prevent the decrease of total antioxidant capacity as well as causing improvement of maximal oxygen uptake (VO2 max) and muscle fatigue (34). Infusion of Nacetylcysteine before and during exercise showed that inhibition of ROS generation could interact with some signaling pathways involved in muscle adaptive response (35). An interesting issue in relation to $\mathrm{N}$-acetyl cysteine supplementation during exercise training is that previous studies focused mainly on its beneficial properties, whereas recent investigations involving cellular pathways, believe it may impair intrinsic muscle responses and recovery (36).

Vitamin E:Vitamin E is the primary chain-breaking antioxidant in cell membranes and other lipid-rich structures such as mitochondria, sarcoplasmic reticulum which limits lipid peroxidation (12). Vitamin $\mathrm{E}$ has an important role in the conversion of superoxide, hydroxyl and lipid peroxyl radicals to less reactive forms (37). Observations in animal models suggest that acute sub-maximal exercise reduces vitamin E concentrations in skeletal muscle and increases requirements for the vitamin (38). Moreover this deficiency increases probable oxidative damage of lysosomal membranes and decline in endurance capacity (39). Vitamin E supplementation in humans reduces oxidative stress, lipid peroxidation and muscle soreness after exercise in some, but not all at of the all studies; for example, in one study, significant reduction of exercise-induced oxidative injury was reported by administration of $800 \mathrm{mg} \alpha$-tocopherol for 48 days (40). In another study, compared to placebo supplementation, with $300 \mathrm{mg} \alpha$-tocopherol in competitive cyclists prevented the increase of serum malondialdehyde (MDA) and creatine kinase (marker of membrane damage) (41). Subudhi et al. reported that vitamin E supplementation following an intense bout of exercise could prevent urinary increase of malondialdehyde (42). Aoi et al. found that vitamin E may reduce the infiltration of neutrophils into the muscle after exercise and significantly attenuate post-exercise increase of muscle myeloperoxidase (43). Keong et al. reported that although tocotrienol-rich palm vitamin E supplementation decreased lipid peroxidation at rest and during exercise training, it however did not enhance endurance running performance or prevent exercise-induced muscle damage (44). In general, most well-controlled studies have not found an ergogenic effect of vitamin E supplementation either on performance during standard exercise tests or cardio respiratory fitness tests $(40,45)$. On the other hand, vitamin $\mathrm{E}$ supplementation could modulate redox-regulated adaptive responses to muscle contractions and consequently disturb optimal muscle function (46).

\subsection{Vitamin $C$}

Vitamin C is a water soluble antioxidant that directly scavenges superoxide, hydroxyl and lipid hydro peroxide radicals, and plays an important role in recycling the vitamin E generated in membranes during oxidative stress (47). Vitamin C has a strong regulatory effect on neutrophils' production of ROS (48). Similar to other antioxidants, the effects of vitamin $C$ supplementation during exercise training are controversial; it was investigated that vitamin $C$ supplementation could reduce muscle damage and delayed-onset muscle soreness; however, a well-controlled study found no beneficial effects on either endurance or strength performance (49). The beneficial effect of vitamin $\mathrm{C}$ during aerobic training may involve neutrophil monocyte accumulation in exercised muscle and secretion of cytokines including IL-1, IL-1 $\beta$ and TNF. There is still controversy regarding the use of combined doses of vitamin $\mathrm{C}$ and vitamin $\mathrm{E}$ for a greater effect on stimulating IL-1 $\beta$ and TNF- $\alpha$ than doses each vitamin alone (40). Some researchers showed that vitamin $\mathrm{E}$ and $\mathrm{C}$ decrease IL- 6 response to exercise by prevent- 
Yavari A et al.

ing the release of IL- 6 from contracting skeletal muscle (40). In one study, six weeks of vitamin E and C supplementation prevented endurance exercise-induced lipid peroxidation but had no affect effect on inflammatory markers (45). Colbert et al. reported that inflammatory markers are lower in older adults with higher levels of exercise and in antioxidant users (multivitamin, vitamin E or C, beta carotene) regardless of exercise level (49). Surprisingly, some researchers such as Teixeira et al. have reported that antioxidant supplementation (vitamin $\mathrm{E}$ and $C, \beta$-carotene, lutein, selenium and magnesium) do not offer protection against exercise-mediated lipid peroxidation and inflammation and may delay muscle recovery (50). On the other hand, Zimmermann et al. who investigated the role of antioxidants on changes in skeletal muscle following endurance training, reported that antioxidant supplementation decreased the activities of antioxidant enzymes (such as xanthine oxidase), with a variable effect of endurance training (40). Recently in a clinical trial conducted in female athletes, four-week supplementation with $250 \mathrm{mg} / \mathrm{d}$ vitamin C could reduce the levels of malondialdehyde and creatin kinase (51). Considering current data, it seems that the more important beneficial outcomes of vitamin $\mathrm{C}$ supplementation in exercise training are attenuation of exercise-induced bronchoconstriction and asthma, as well as decrease in muscle damage $(52,53)$. However administration of nonphysiological doses of vitamin $\mathrm{C}$ in healthy athletes is not recommended because of a possibility of its impairing favorable adaptation of regular exercise including redox homeostasis (54).

\subsection{Plant-Food Polyphenols}

Polyphenols are natural phytochemical compounds including phenolic acids, flavonoids, stilbenes, lignans and polymeric lignans identified in whole plant foods. Dietary polyphenols have potent antioxidant properties and could modulate some important cell signaling pathways NF-kB, mitogen-activated protein kinases (MAPK), and nuclear factor erythroid 2 related factor 2 (Nrf2) (55, 56). The most known properties of polyphenols are scavenging of superoxide, hydroxyl and peroxyl radicals, and also inhibition of lipid peroxidation, metal iron-mediated radical formation and preventing radical mediated depletion of vitamin E and $\beta$-carotene (57). Some protective effects against exercise-induced oxidative stress have been demonstrated by polyphenol supplements (58). Administration of $150 \mathrm{~g} / \mathrm{d}$ blueberries, rich sources of polyphenols, significantly decreased serum levels of free radicals in athletes in a hot environment (59). Chang et al. reported that consuming a high-polyphenol diet (purple sweet potato leaves) for 7 days can modulate antioxidative status and decrease exercise-induced oxidative damage and pro-inflammatory cytokine secretion (60). In another study, combination of polyphenols (catechins, chlorogenic acid, ellagic acid and quercetin) enhanced the swimming performance of rats. In this study polyphenols increased the concentration of ATP and glycogen in muscle and reduced MDA levels in the liver, muscle and blood; activities of lactic dehydrogenase and creatine phosphokinase were also decreased (61). Oligomeric proanthcyanidins found in grapes, cocoa and apples could enhance performance of sportsmen by a protective action during physical exercise (62). More interestingly, against other antioxidants that could impair mitochondrial redox pathways involved in exercise adaption, polyphenols induce mitochondrial adaptive redox pathways (62). So polyphenols compared to other antioxidants may be considered as appropriate supplement for exercise training (63). Despite in vitro and animal models indicating beneficial effects of polyphenols on exercise-induced oxidative stress, muscle damage and exercise performance, it seems more studies in humans are needed to confirm these results (64).

\subsection{Other Antioxidant Supplements}

Carotenoids (e.g. $\beta$-carotenes) lipid-soluble antioxidants located primarily in biological membranes, could can reduce lipid peroxidation; studies show that astaxanthin, a member of the carotenoid family, and a dark-red pigment found in the marine world of algae and aquatic animals such as salmon, red sea bream as well as in birds such as flamingo and quail, has potential health-promoting effects in the exercise-induced fatigue (65).

Ubiquinones are lipid-soluble quinon derivatives, which in reduced form act as antioxidants; ubiquinones react with ROS to prevent lipid peroxidation, and have an important role in the recycling of vitamin E (66). Coenzyme Q10 is the predominant form of ubiquinones in humans, which is found in soybean oil, meats, fish, nuts, wheat germ and vegetables (67). Current data regarding supplementation with ubiquinones during exercise training are inconsistent; a positive relationship between exercise capacity and concentration of coenzyme Q10 in physically active males was reported but some studies have failed to demonstrate the claimed ergogenic properties for ubiquinones, and some studies have even shown impaired performance following high-intensity and endurance tests after supplementation with coenzyme Q10 (6). Diaz-Castro et al. reported that CoQ-10 supplementations before strenuous exercise decreased oxidative stress parameters including 8-hydroxy-2 dexyguanosine and isoprostanes levels and prevented overexpression of TNF- $\alpha$ after exercise (66). In another study of young swimmers, 12 days of CoQ-10 supplementation reduced MDA, nitric oxide and protein hydroperoxide, while increasing maximal treadmill time (68). One study showed significant improved indexes of physical performance, while another did not report any improvement in aerobic capacity following Q10 supplementation (6).

$\alpha$-Lipoic acid is an endogenous thiol and co-factor of $\alpha$-dehydrogenase complexes that are reduced to dihydro- 
Yavari A et al.

lipoic acid (DHLA; a potent antioxidant against all major oxy radical species) following dietary supplementation; DHLA is an important agent in recycling vitamin C during oxidative stress and can be an effective glutathione substitute (69). Supplementation with $\alpha$-lipoic acid enhances muscle phosphocreatine levels and muscle total creatine concentrations and consequently has a potential enhancing effect on short-term exercise (6). However its effects on isokinetic exercise performance are unknown. A recent study suggests that vitamin $\mathrm{E}$ and $\alpha$-lipoic acid supplementation may in fact suppress skeletal muscle mitochondrial biogenesis, regardless of training status (70). Some studies have shown the benefits of lipoic acid or vitamin E supplementations in endurance trained horses (71).

Nitric oxide (NO) has been implicated in the improvement of exercise capacity through vascular smooth muscle relaxation in both coronary and skeletal muscle arteries as well as via independent mechanisms. Endothelial nitric oxide synthase (eNOS) uses the amino acid L-arginine as a substrate to synthesize nitric oxide (NO). On the other hand, antioxidants may prevent nitric oxide inactivation by oxygen free radicals (6). Chen et al. investigated the effects of L-arginine and antioxidant supplements on exercise performance in elderly male cyclists and reported that this intervention has a potential role in improving exercise performance in the elderly (72).

Spirulina is a blue-green alga that seems to exert antioxidant properties, which are attributed to molecules such as phytocyanin, $\beta$-carotene, tocopherol, $\gamma$-linolenic acid and phenolic compounds; it has also shown a preventive effect against the skeletal damage under exercise induced oxidative stress (73). Anthocyanins (e.g. chokeberry juice) limit the exercise-induced oxidative damage to red blood cells, most probably by enhancing the endogenous antioxidant defense system (74).Superoxide dismutase (SOD), a key enzyme that catalyzes the reduction of superoxide anions to less reactive hydrogen peroxide, is found in some plants but has limited use due to the inactivation of the enzyme in the gastrointestinal tract. Recently an original and modified vegetable formula made from SOD-rich melon extract has been developed as an oral route agent; this product has been investigated and seems to promote antioxidant status in professional rowers without effect on oxidative damage induced by exhaustive exercise (75).

\subsection{Trace Minerals}

The main minerals involved in antioxidant-related functions include copper ( $\mathrm{Cu})$, zinc $(\mathrm{Zn})$, iron (Fe), selenium (Se) and manganese (Mn); their antioxidant effects contribute to the action as co-factors for antioxidant enzymes (6). Copper and zinc as co-factors for $\mathrm{Cu} \mathrm{Zn}$-superoxide dismutase which is responsible for eliminating superoxide radicals. Iron is an essential co-factor in the antioxidant enzyme catalase. Catalase removes hydrogen peroxide from cells (6). Increasing dietary or supplemental Fe can improve performance and may help prevent decreases in ferritin associated with exercise. Moderatelevel supplementation prevented a decrease in serum ferritin in competitive swimmers (40). Selenium (Se) is a co-factor for the antioxidant enzyme glutathione peroxidase, which is responsible for removing hydrogen peroxide and other organic hydroperoxide from the cell; Results of the study of Akil et al. indicate that acute swimming exercise in rats increased the lipid peroxidation in the brain tissue of rats, while selenium supplementation prevented the free radical formation by enhancing the antioxidant activity (76).

Manganese (Mn) is co-factor for manganese-superoxide dismutase, which has a role in eliminating of superoxide radicals produced by oxidative phosphorylation (6). Trials on Magnesium (Mg) supplementation in athletes have shown different findings. Some studies have reported a considerable reduction in total serum creatin kinase, serum lactate concentration as well as improvement of cardio-respiratory function after $\mathrm{Mg}$ supplementation (6).

Current data suggest that Mg supplementation does not affect performance when serum $\mathrm{Mg}$ is within the range of normal values, but may improve performance when marginal or clinical Mg deficiency is present (40). Zinc is an important co-factor in a range of immune functions; supplementation with a combination of $\mathrm{Zn} /$ $\mathrm{Cu}$ prevented an increase in the capacity of neutrophils to produce ROS in vitro immediately after exercise (15). Polat et al. showed that the combined effects of exercise and zinc supplement have a positive effect on the hematological parameters of athletes that may lead to better performance and increased endurance (77).

\subsection{Exercise-Induced Oxidative Stress and Dietary Recommendation}

Although a balanced vegetarian diet high in antioxidantrich foods has been proposed as a dietary recommendation to enhance endogenous antioxidative capacity and attenuate exercise-induced oxidative stress, studies on vegetarian athletes is lacking and there is no sufficient evidence to support this hypothesis (78). Limited investigations have reported effects of antioxidant-rich foods on exercise-induced oxidative stress; consumption of a diet rich in Allium vegetables (Allium sativum, Allium cepa, Allium fistulosum or Allium tuberosum) before and after exercise training increased the ratio of reduced glutathione to oxidized glutathione in rat models (79). A restricted-antioxidant-rich food diet for 2 weeks compared to habitual diet, increased serum levels of F2-isoprostanes (oxidative stress marker) following sub-maximal exercise by $38 \%$, exhaustive exercise by $45 \%$, and one hour recovery by $31 \%$ in trained athletes. These findings indicate that athletes require higher intake of natural antioxidants especially antioxidant-rich foods (80). 


\section{Conclusions}

It seems that the best recommendation regarding antioxidants and exercise is having a balanced diet rich in natural antioxidants and phytochemicals. Regular consumption of various fresh fruits and vegetables, whole grains, legumes and beans, sprouts and seeds is an effective and safe way to meet all antioxidants requirements in physically active persons and the athletes.

The increase in production of free radicals with intense physical exercise can exceed the capacity of the antioxidant defense systems in the body and induce oxidative conditions; currently however both positive and negative aspects of ROS generation in sport performance are considered. Despite remarkable evidence of the positive effects of various vitamins and supplements in improvement of unfavorable imbalance between oxidative reactions and antioxidant equilibrium, controversial data are observed in literature; some investigators even believe that supplementation with antioxidants prevent health-promoting effects of physical exercise and may be harmful in humans or may delay muscle recovery; antioxidant supplementations may also block the positive effects of exercise on improved insulin sensitivity. Overall, there is insufficient data supporting the effectiveness of antioxidant supplements to prevent the probably probable damages of strenuous exercise, particularly the improvement of physical exercise performance.

\section{Acknowledgements}

The authors wish to acknowledge Ms Niloofar Shiva for critical editing of English grammar and syntax of the manuscript. None of the authors had any personal or financial conflicts of interest.

\section{References}

1. Giustarini D, Dalle-Donne I, Tsikas D, Rossi R. Oxidative stress and human diseases: Origin, link, measurement, mechanisms, and biomarkers. Crit Rev Clin Lab Sci. 2009;46(5-6):241-81.

2. Otani H. Oxidative stress as pathogenesis of cardiovascular risk associated with metabolic syndrome. Antioxid Redox Signal. 2011;15(7):1911-26.

3. Powers SK, Nelson WB, Hudson MB. Exercise-induced oxidative stress in humans: cause and consequences. Free Radic Biol Med. 2011;51(5):942-50.

4. Powers SK, Jackson MJ. Exercise-induced oxidative stress: cellular mechanisms and impact on muscle force production. Physiol Rev. 2008;88(4):1243-76.

5. Kinnunen S, Atalay M, Hyyppa S, Lehmuskero A, Hanninen O, Oksala N. Effects of prolonged exercise on oxidative stress and antioxidant defense in endurance horse. J Sports Sci Med. 2005;4(4):415-21.

6. Powers SK, DeRuisseau KC, Quindry J, Hamilton KL. Dietary antioxidants and exercise. J Sports Sci. 2004;22(1):81-94.

7. Powers SK, Talbert EE, Adhihetty PJ. Reactive oxygen and nitrogen species as intracellular signals in skeletal muscle.J Physiol. 2011;589(Pt 9):2129-38.

8. Jackson MJ. Free radicals generated by contracting muscle: byproducts of metabolism or key regulators of muscle function? Free Radic Biol Med. 2008;44(2):132-41.

9. Goldstein BJ, Mahadev K, Wu X. Redox paradox: insulin action is facilitated by insulin-stimulated reactive oxygen species with multiple potential signaling targets. Diabetes. 2005;54(2):311-21.

10. Ristow $M$, Zarse $K$, Oberbach A, Kloting N, Birringer $M$ Kiehntopf M, et al. Antioxidants prevent health-promoting effects of physical exercise in humans. Proc Natl Acad Sci U S A 2009;106(21):8665-70.

11. Ji LL. Exercise-induced modulation of antioxidant defense. Ann $N$ YAcad Sci. 2002;959:82-92.

12. Ji LL, Gomez-Cabrera MC, Vina J. Exercise and hormesis: activation of cellular antioxidant signaling pathway. Ann N Y Acad Sci. 2006;1067:425-35.

13. Dionyssiotis Y. . Topics in Paraplegia. Guolin L editor. : InTech; 2013.

14. Rahal A, Kumar A, Singh V, Yadav B, Tiwari R, Chakraborty S, et al. Oxidative stress, prooxidants, and antioxidants: the interplay. Biomed Res Int. 2014;2014:761264.

15. Spector A. Review: Oxidative stress and disease. J Ocul Pharmacol Ther. 2000;16(2):193-201.

16. Kelkar G, Subhadra K, Chengappa RK. Effect of antioxidant sup plementation on hematological parameters, oxidative stress and performance of Indian athletes. J. Hum.Ecol. 2008;24(3):209-13.

17. Peake J, Suzuki K. Neutrophil activation, antioxidant supplements and exercise-induced oxidative stress. Exerc Immunol Rev. 2004;10:129-41.

18. Lambertucci RH, Levada-Pires AC, Rossoni LV, Curi R, Pithon-Curi TC. Effects of aerobic exercise training on antioxidant enzyme activities and mRNA levels in soleus muscle from young and aged rats. Mech Ageing Dev. 2007;128(3):267-75.

19. Cooper CE, Vollaard NB, Choueiri T, Wilson MT. Exercise, free radicals and oxidative stress. Biochem Soc Trans. 2002;30(2):280-5.

20. Belviranlı M, Gökbel H. Acute exercise induced oxidative stress and antioxidant changes. Eur J Gen Med. 2006;3(3):126-31.

21. Liu J, Yeo HC, Overvik-Douki E, Hagen T, Doniger SJ, Chyu DW, et al. Chronically and acutely exercised rats: biomarkers of oxidative stress and endogenous antioxidants. J Appl Physiol (1985). 2000;89(1):21-8.

22. Ji LL. Antioxidant signaling in skeletal muscle: a brief review. Exp Gerontol. 2007;42(7):582-93.

23. Elokda AS, Nielsen DH. Effects of exercise training on the glutathione antioxidant system. Eur I Cardiovasc Prev Rehabil. 2007;14(5):630-7.

24. Terblanche SE. The effects of exhaustive exercise on the activity levels of catalase in various tissues of male and female rats. Cell Biol Int. 2000;23(11):749-53.

25. Psaltopoulou T, Panagiotakos DB, Pitsavos C, Chrysochoou C, Detopoulou P, Skoumas J, et al. Dietary antioxidant capacity is inversely associated with diabetes biomarkers: the ATTICA study. Nutr Metab Cardiovasc Dis. 2011;21(8):561-7.

26. Bahadoran Z, Golzarand M, Mirmiran P, Shiva N, Azizi F. Dietary total antioxidant capacity and the occurrence of metabolic syndrome and its components after a 3-year follow-up in adults Tehran Lipid and Glucose Study. Nutr Metab (Lond). 2012;9(1):70.

27. Bahadoran Z, Mirmiran P, Hosseinpanah F, Hedayati M, Hosseinpour-Niazi S, Azizi F. Broccoli sprouts reduce oxidative stress in type 2 diabetes: a randomized double-blind clinical trial. Eur Clin Nutr. 2011;65(8):972-7.

28. Mirmiran P, Noori N, Zavareh MB, Azizi F. Fruit and vegetable consumption and risk factors for cardiovascular disease. Metabolism. 2009;58(4):460-8.

29. Sohrab G, Hosseinpour-Niazi S, Hejazi J, Yuzbashian E, Mirmiran P, Azizi F. Dietary polyphenols and metabolic syndrome among Iranian adults. Int J Food Sci Nutr. 2013;64(6):661-7.

30. Brisswalter J, Louis J. Vitamin supplementation benefits in mas ter athletes. Sports Med. 2014;44(3):311-8.

31. Leeuwenburgh C, Heinecke JW. Oxidative stress and antioxidants in exercise. Curr Med Chem. 2001;8(7):829-38.

32. Lushchak VI. Glutathione homeostasis and functions: potential targets for medical interventions. JAmino Acids. 2012;2012:736837.

33. Whillier S, Raftos JE, Chapman B, Kuchel PW. Role of N-acetylcysteine and cystine in glutathione synthesis in human erythrocytes. Redox Rep. 2009;14(3):115-24.

34. Huupponen MR, Makinen LH, Hyvonen PM, Sen CK, Rankinen $\mathrm{T}$, Vaisanen $\mathrm{S}$, et al. The effect of $\mathrm{N}$-acetylcysteine on exercise- 
Yavari A et al.

induced priming of human neutrophils. A chemiluminescence study. Int J Sports Med. 1995;16(6):399-403.

35. Leelarungrayub D, Khansuwan R, Pothongsunun P, Klaphajone J $\mathrm{N}$-acetylcysteine supplementation controls total antioxidant capacity, creatine kinase, lactate, and tumor necrotic factor-alpha against oxidative stress induced by graded exercise in sedentary men. Oxid Med Cell Longev. 2011;2011:329643.

36. Petersen AC, McKenna MJ, Medved I, Murphy KT, Brown MJ, Della Gatta P, et al. Infusion with the antioxidant N-acetylcysteine attenuates early adaptive responses to exercise in human skeletal muscle. Acta Physiol (Oxf). 2012;204(3):382-92.

37. Michailidis Y, Karagounis LG, Terzis G, Jamurtas AZ, Spengos K, Tsoukas D, et al. Thiol-based antioxidant supplementation alters human skeletal muscle signaling and attenuates its inflammatory response and recovery after intense eccentric exercise. Am Clin Nutr. 2013;98(1):233-45.

38. Traber MG, Atkinson J. Vitamin E, antioxidant and nothing more Free Radic Biol Med. 2007;43(1):4-15.

39. Swift JJ, Kehrer JP, Seiler KS, Starnes JW. Vitamin E concentration in rat skeletal muscle and liver after exercise. Int J Sport Nutr. 1998;8(2):105-12.

40. Zimmermann MB. Vitamin and mineral supplementation and exercise performance. Schweiz Z Med Traumatol. 2003;51(1):53-7.

41. Rokitzki L, Logemann E, Huber G, Keck E, Keul J. alpha-Tocopherol supplementation in racing cyclists during extreme endurance training. Int J Sport Nutr. 1994;4(3):253-64.

42. Subudhi AW, Mattson JP. Effects of Antioxidant Supplementation on Oxidative Stress in Trained Cyclists. USANA Health Sci. 2000.

43. Aoi W, Naito Y, Takanami Y, Kawai Y, Sakuma K, Ichikawa H, et al. Oxidative stress and delayed-onset muscle damage after exercise. Free Radic Biol Med. 2004;37(4):480-7.

44. Keong CC, Singh HJ, Singh R. Effects of palm vitamin e supplementation on exercise-induced oxidative stress and endurance performance in the heat. J Sports Sci Med. 2006;5(4):629-39.

45. Bloomer RJ, Falvo MJ, Schilling BK, Smith WA. Prior exercise and antioxidant supplementation: effect on oxidative stress and muscle injury. J Int Soc Sports Nutr. 2007;4:9.

46. Jackson MJ, Khassaf M, Vasilaki A, McArdle F, McArdle A. Vitamin $\mathrm{E}$ and the oxidative stress of exercise. Ann $N$ Y Acad Sci. 2004;1031:158-68.

47. Kojo S. Vitamin C: basic metabolism and its function as an index of oxidative stress. Curr Med Chem. 2004;11(8):1041-64.

48. Dwenger A, Funck M, Lueken B, Schweitzer G, Lehmann U. Effect of ascorbic acid on neutrophil functions and hypoxanthine/xanthine oxidase-generated, oxygen-derived radicals. EurJ Clin Chem Clin Biochem.1992;30(4):187-91.

49. Colbert LH, Visser M, Simonsick EM, Tracy RP, Newman AB, Kritchevsky SB, et al. Physical activity, exercise, and inflammatory markers in older adults: findings from the Health, Aging and Body Composition Study.J Am Geriatr Soc. 2004;52(7):1098-104.

50. Teixeira VH, Valente HF, Casal SI, Marques AF, Moreira PA. Antioxidants do not prevent postexercise peroxidation and may delay muscle recovery. Med Sci Sports Exerc. 2009:1752-60.

51. Taghiyar M, Darvishi L, Askari G, Feizi A, Hariri M, Mashhadi NS et al. The effect of vitamin $C$ and e supplementation on muscle damage and oxidative stress in female athletes: a clinical trial. Int JPrev Med. 2013;4(Suppl 1):S16-23.

52. Askari G, Ghiasvand R, Karimian J, Feizi A, Paknahad Z, Sharifirad $\mathrm{G}$, et al. Does quercetin and vitamin $\mathrm{C}$ improve exercise performance, muscle damage, and body composition in male athletes? JRes Med Sci. 2012;17(4):328-31.

53. Hemila H. Vitamin C may alleviate exercise-induced bronchoconstriction: a meta-analysis. BMJ Open. 2013;3(6).

54. Nikolaidis MG, Kerksick CM, Lamprecht M, McAnulty SR. Does vitamin $\mathrm{C}$ and $\mathrm{E}$ supplementation impair the favorable adaptations of regular exercise? Oxid Med Cell Longev. 2012;2012:707941.

55. Pandey KB, Rizvi SI. Plant polyphenols as dietary antioxidants in human health and disease. Oxid Med Cell Longev. 2009;2(5):270-8.

56. Han X, Shen T, Lou H. Dietary Polyphenols and Their Biological Significance. Int J Mol Sci. 2007;8(9):950-88.

57. Urquiaga I, Leighton F. Plant polyphenol antioxidants and oxidative stress. Biol Res. 2000;33(2):55-64.
58. Morillas-Ruiz JM, Villegas Garcia JA, Lopez FJ, Vidal-Guevara ML, Zafrilla P. Effects of polyphenolic antioxidants on exercise-induced oxidative stress. Clin Nutr. 2006;25(3):444-53.

59. McAnulty SR, McAnulty LS, Nieman DC, Dumke CL, Morrow JD, Utter AC, et al. Consumption of blueberry polyphenols reduces exercise-induced oxidative stress compared to vitamin C. Nutr Res . 2004;24(3):209-21.

60. Chang WH, Hu SP, Huang YF, Yeh TS, Liu JF. Effect of purple sweet potato leaves consumption on exercise-induced oxidative stress and IL-6 and HSP72 levels. J Appl Physiol . 2010;109(6):1710-5.

61. Swamy MS, Sivanna N, Tamatam A, Khanum F. Effect of poly phenols in enhancing the swimming capacity of rats. (FFHD). 2011;1(11):482-91.

62. Lafay S, Jan C, Nardon K, Lemaire B, Ibarra A, Roller M, et al. Grape extract improves antioxidant status and physical performance in elite male athletes. J Sports Sci Med. 2009;8(3):468-80.

63. Badria FA. Evidence-based Strategies in Herbal Medicine, Psychiatric Disorders and Emergency Medicine. E. Stevenson editor.:Intech;2012.

64. Malaguti M, Angeloni C, Hrelia S. Polyphenols in exercise performance and prevention of exercise-induced muscle damage. Oxid Med Cell Longev. 2013;2013:825928.

65. Dhankhar J, Kadian SS, Sharma A. Astaxanthin: A potential carotenoid. Int JPharmaceut Sci Res. 2012;3:1246-59.

66. Diaz-Castro J, Guisado R, Kajarabille N, Garcia C, Guisado IM, de Teresa C, et al. Coenzyme Q(10) supplementation ameliorates inflammatory signaling and oxidative stress associated with strenuous exercise. Eur J Nutr. 2012;51(7):791-9.

67. Crane FL. Discovery of ubiquinone (coenzyme Q) and an overview of function. Mitochondrion. 2007;7 Suppl:S2-7.

68. Leelarungrayub D, Sawattikanon N, Klaphajone J, Pothongsunan P, Bloomer RJ. Coenzyme Q10 supplementation decreases oxidative stress and improves physical performance in young swimmers; a pilot study. The Open Sports Med J. 2010;4:1-8.

69. Shay KP, Moreau RF, Smith EJ, Smith AR, Hagen TM. Alpha-lipoic acid as a dietary supplement: molecular mechanisms and therapeutic potential. Biochim Biophys Acta. 2009;1790(10):1149-60.

70. Lappalainen Z. Effect of alpha-lipoic acid and time-of-day on interleukin-6 response to exhaustive exercise in humans. Afr $\mathrm{J}$ Pharm Pharacol. 2009;5(1):42-7.

71. Williams CA. Antioxidant supplementation to the exercising horse. Revista Brasileira de Zootecnia. 2010;39:145-50.

72. Chen S, Kim W, Henning SM, Carpenter CL, Li Z. Arginine and antioxidant supplement on performance in elderly male cyclists: a randomized controlled trial. J Int Soc Sports Nutr. 2010;7:13.

73. Chu WL, Lim YW, Radhakrishnan AK, Lim PE. Protective effect of aqueous extract from Spirulina platensis against cell death induced by free radicals. BMC Complement Altern Med. 2010;10:53.

74. Pilaczynska-Szczesniak L, Skarpanska-Steinborn A, Deskur E, Basta P, Horoszkiewicz-Hassan M. The influence of chokeberry juice supplementation on the reduction of oxidative stress resulting from an incremental rowing ergometer exercise. Int J Sport Nutr Exerc Metab. 2005;15(1):48-58.

75. Skarpanska-Stejnborn A, Pilaczynska-Szczesniak L, Basta P, DeskurSmielecka E, Woitas-Slubowska D, Adach Z. Effects of oral supplementation with plant superoxide dismutase extract on selected redox parameters and an inflammatory marker in a 2,000-m rowing-ergometer test. Int J Sport Nutr Exerc Metab. 2011;21(2):124-34.

76. Akil M, Bicer M, Menevse E, Baltaci AK, Mogulkoc R. Selenium supplementation prevents lipid peroxidation caused by arduous exercise in rat brain tissue. Bratisl Lek Listy. 2011;112(6):314-7.

77. Polat Y. Effects of zinc supplementation on hematological parameters of high performance athletes. African Journal of Pharmacy and Pharma cology. 2011;5(12):1436-40.

78. Trapp D, Knez W, Sinclair W. Could a vegetarian diet reduce exercise-induced oxidative stress? A review of the literature. J Sports Sci. 2010;28(12):1261-8

79. Choi EY, Cho YO. Allium vegetable diet can reduce the exerciseinduced oxidative stress but does not alter plasma cholesterol profile in rats. Ann Nutr Metab. 2006;50(2):132-8.

80. Watson TA, Callister R, Taylor R, Sibbritt D, MacDonald-Wicks LK, Garg ML. Antioxidant restricted diet increases oxidative stress during acute exhaustive exercise. Asia Pac J Clin Nutr. 2002;12:S9. 